\title{
Is Neural Processing of Negative Stimuli Altered in Addiction Independent of Drug Effects? Findings From Drug-Naïve Youth with Internet Gaming Disorder
}

\author{
Sarah W Yip', James J Gross', Megha Chawla', Shan-Shan Ma ${ }^{3}$, Xing-Hui Shi ${ }^{3}$, Lu Liu', Yuan-Wei Yao ${ }^{3}$, \\ Lei Zhu', Patrick D Worhunsky' and Jintao Zhang*,3,4 \\ 'Department of Psychiatry, Yale School of Medicine, New Haven, CT, USA; '2Department of Psychology, Stanford University, Stanford, CA, USA; \\ ${ }^{3}$ State Key Laboratory of Cognitive Neuroscience and Learning and IDG/McGovern Institute for Brain Research, Beijing Normal University, Beijing, \\ China; ${ }^{4}$ Institute of Developmental Psychology, Beijing Normal University, Beijing, China
}

\begin{abstract}
Difficulties in emotion regulation are commonly reported among individuals with alcohol and drug addictions and contribute to the acquisition and maintenance of addictive behaviors. Alterations in neural processing of negative affective stimuli have further been demonstrated among individuals with addictions. However, it is unclear whether these alterations are a general feature of addictions or are a result of prolonged exposure to drugs of abuse. To test the hypothesis of altered negative affect processing independent of drug effects, this study assessed neural function among drug-naïv youth with a behavioral addiction — Internet gaming disorder (IGD). Fifty-six young adults (28 with IGD, 28 matched controls) participated in $\mathrm{MRR}$ scanning during performance of a well-validated emotion regulation task. Between-group differences in neural activity during task performance were assessed using a whole-brain, mixed-effects ANOVA with correction for multiple comparisons at currently recommended thresholds (voxel-level $p<0.00 \mathrm{I}$, pFWE $<0.05$ ). Compared to controls, youth with IGD exhibited significantly blunted neural responses within distributed subcortical and cortical regions including the striatum, insula, lateral prefrontal cortex and anterior cingulate in response to negative affective cues, as well as during emotion regulation. Independent component analysis (ICA) further identified between-group differences in engagement of a fronto-cingulo-parietal network, involving decreased engagement in IGD youth relative to controls. Study findings are largely consistent with those from prior neuroimaging studies in substance-use disorders, thus raising the possibility that neural processing of negative affect may be blunted across drug and behavioral addictions independent of acute or chronic drug effects.
\end{abstract}

Neuropsychopharmacology (2018) 43, I364-1372; doi:10.1038/npp.2017.283; published online 20 December 2017

\section{INTRODUCTION}

Depressive symptoms, increased negative affect and difficulties in emotion regulation are common among individuals with alcohol and drug-use disorders (DUDs) and are increasingly recognized as a part of the phenomenology of addictions (Fox et al, 2007; Volkow et al, 2016). In nonaddicted individuals, depressive symptoms and difficulties in emotion regulation are typically accompanied by heightened engagement of limbic regions (eg, amygdala, insula, and striatum), and decreased engagement of cortical regions (eg, ventral and dorsolateral prefrontal cortex) (Etkin and Wager, 2007; Wilcox et al, 2016). However, neuroimaging data generally suggest decreased neural activity within both limbic

*Correspondence: Dr J Zhang, State Key Laboratory of Cognitive Neuroscience and Learning and IDG/McGovern Institute for Brain Research, No. 19 XiejieKouWai Street, Haidian Strict, Beijing 100875 , China, Tel: +86 I058800728, Fax: +86I5010233046,

E-mail: zhangiintao@bnu.edu.cn

Received 29 June 2017; revised 5 November 2017; accepted 8 November 2017; accepted article preview online 20 November 2017 and cortical regions in response to stress and negative affect cues among individuals with DUDs (Seo et al, 2013; AlbeinUrios et al, 2014; Wilcox et al, 2016). Together, existing data therefore suggest differing neural substrates of negative affect processing and regulation among individuals with addictions $v s$ other psychiatric disorders, which could either be a consequence of prolonged drug-use or a core feature of addictions more generally (or a combination of the two). To test the hypothesis of alterations in negative affect processing independent of the exogenous effects of drugs of abuse, this study assesses neural function during emotion regulation task performance among drug-naïve youth with a behavioral addiction-Internet gaming disorder (IGD). We chose to study IGD rather than gambling disorder because of the relatively lower rates of substance-use generally reported in this population (Barnes et al, 2009; Yip et al, 2011; Peters et al, 2015; Mihara and Higuchi, 2017).

IGD is a behavioral addiction included in the current DSM-5 as a condition for further study (American Psychiatric Association Committee on Nomenclature and Statistics, 2013). As with other behavioral addictions, the 
diagnostic criteria for IGD are based on those for DUDs and include tolerance, gaming to escape negative moods, loss of control and continued gaming despite negative consequences (American Psychiatric Association Committee on Nomenclature and Statistics, 2013; Petry et al, 2014). As with DUDs, IGD is clinically characterized by alterations in reward processing, high rates of impulsivity and depressive symptoms and significant distress related to the behavior/ drug (Lehenbauer-Baum et al, 2015; Petry et al, 2015; Yao et al, 2015). While the neurobiology of IGD remains an area of ongoing investigation, extant data further indicate biological similarities with DUDs, including cross-sectional data suggesting a transition from ventral to dorsal striatal processing of addiction cues (Liu et al, 2017; Weinstein et al, 2017). However, no prior study has assessed neural processing of negative affect among individuals with IGD.

Here, we compare neural responses between drug-naïve young adults with and without IGD during performance of a well-validated emotion regulation task (Ochsner et al, 2002) using both whole-brain general-linear-model (GLM) and independent component analysis (ICA) approaches. ICA is a data-driven, network-based approach for studying temporally coherent, functionally-integrated networks of neural activity (Calhoun et al, 2001a, 2001b; Meda et al, 2012; Smith et al, 2012). This approach is sensitive to patterns of neural activity that may not be detected using traditional GLMbased approaches, and thus can provide complimentary information to a GLM-based approach, even within the context of the same brain region (Xu et al, 2013a,b, 2016). Given the centrality of the amygdala to affective processing (Ochsner et al, 2012; Etkin et al, 2015), we further conducted a region-of-interest (ROI) analysis focusing on this structure.

Based on prior findings in adults with cocaine-use disorder (Albein-Urios et al, 2014), we hypothesized significantly decreased activity among IGD youth within brain regions including the thalamus, amygdala, striatum and dorsal and lateral prefrontal cortex (PFC) during both passive emotion processing and regulation ( $v s$ neutral). In other words, we hypothesized that individuals with IGD would fail to show normative responses to negative affective stimuli, and that this would be present during both passive emotion processing and during emotion regulation. Based on prior connectivity findings (Albein-Urios et al, 2014), we further anticipated decreased engagement of cortical inhibitory control networks among IGD youth, as indexed using ICA.

\section{MATERIALS AND METHODS}

\section{Participants}

Participants were recruited from Beijing, China via advertisements within Beijing Normal University and the surrounding area. Given the disproportionate incidence of IGD in young-adult males, only male participants (ages 1826) were included in this study (Petry et al, 2015; Rehbein et al, 2015). Potential participants were screened using the Chinese version of the MINI International Neuropsychiatric Interview (Sheehan et al, 1998; Si et al, 2009) and participated in clinical interviews to determine the presence or absence of IGD, based on DSM-5 suggested criteria. Specifically, participants were individually screened for IGD in one-on-one interviews with trained members of the research staff. These interviews focused on the nine criteria listed in the DSM-5 and participants were asked to answer these nine items with 'yes' or 'no', those who have five or more 'yes' answers were classified as IGD.

Exclusion criteria for all participants included use of illicit drugs (any prior exposure), current or past Axis I disorders (excluding IGD for the IGD group) and any history of head trauma resulting in loss of consciousness or other contraindication to MRI scanning (eg, claustrophobia). Additional exclusion criteria (eg, risky drinking) are described in Supplementary Materials. Inclusionary criteria for the IGD group included a DSM-5 diagnosis of IGD (American Psychiatric Association, 2013), a score $\geqslant 50$ or more on a revised version of Young's online Internet addiction test (IAT) (Young, 2009), and gaming online for $>20 \mathrm{~h}$ per week for a minimum of 1 year. Inclusionary criteria for $\mathrm{HCs}$ included an IAT score $<50$ and gaming online for $<2 \mathrm{~h}$ per week.

Fifty-six young adults (28 with IGD, 28 controls) participated in fMRI scanning during emotion regulation task performance. Imaging data from four individuals with IGD and five control participants were excluded due to excessive motion or missing in-scanner data resulting in a final sample of 47 young adults (24 with IGD, 23 controls). Rates of exclusion did not differ between participant groups $\left(x^{2}=0.13, p=0.72\right)$. As shown in Table 1 , IGD and HC

Table I Demographic and Clinical Characteristics of Participants Included in $\mathrm{AMRI}$ Analyses $(n=47)$

\begin{tabular}{|c|c|c|c|c|c|}
\hline & Internet gaming $(n=24)$ & Healthy control $(n=23)$ & $t / x^{2}$ & $p$ & df \\
\hline Tobacco smoker $(n, \%)^{\mathrm{a}}$ & $2(8.7)$ & $0(0.0)$ & 2.09 & 0.49 & 46 \\
\hline Any alcohol use (n, \%) & $6(26.1)$ & II (47.8) & 2.33 & 0.127 & 46 \\
\hline Age (mean, SD) & $22.13(1.45)$ & $22.00(1.81)$ & 0.26 & 0.795 & 45 \\
\hline Chen Internet addiction score & $70.13(10.48)$ & $35.04(10.62)$ & 11.40 & $<0.001$ & 45 \\
\hline Barratt impulsiveness scale & $74.54(9.36)$ & $67.04(7.60)$ & 3.01 & 0.004 & 45 \\
\hline Beck anxiety inventory & $5.29(5.42)$ & $1.35(1.61)$ & 3.35 & 0.002 & 45 \\
\hline AUDIT ${ }^{\mathrm{a}}$ & $0.91(1.76)$ & $1.26(1.91)$ & 0.41 & 0.524 & 44 \\
\hline FTND $^{\mathrm{a}}$ & $0.17(0.65)$ & $0.00(0.00)$ & 1.65 & 0.206 & 44 \\
\hline
\end{tabular}

Abbreviations: AUDIT: alcohol use disorder identification test; FTND, Fagerstöm test for nicotine dependence.

${ }^{\mathrm{a} D a t a}$ missing for I IGD participant. 
participants were well-matched for age, smoking status, and alcohol use. Consistent with prior work in different cohorts (Zhang et al, 2016b, 2016c; Liu et al, 2017), and with the findings from DUDs, IGD participants scored significantly higher on self-report indices of impulsivity (Patton et al, 1995), depression (Beck et al, 1996), and anxiety (Beck et al, 1988) ( $p$ 's<0.005; details in Table 1).

\section{Emotion Regulation Task}

During emotion regulation task performance (Ochsner et al, 2002), participants are presented with stimuli from the International Affective Picture System that have been previously validated as either negative or neutral. Prior to the presentation of each image, participants are instructed to either respond naturally and attend to the stimulus while maintaining their emotional response ('LOOK'), or to decrease their emotional response via cognitive reappraisal of the stimulus ('DECREASE'). Neutral images are always preceded by a LOOK instruction, whereas negative images may be preceded by either the LOOK or DECREASE instruction. The task therefore includes three trial types: (i) neutral image with instruction to respond naturally (neutral trials; $n=15$ ); (ii) negative image with instruction to respond naturally (look trials; $n=15$ ); and (iii) negative image with instruction to regulate emotional response via cognitive reappraisal (decrease trials; $n=15$ ). Negative images for the look and decrease trials were well-matched on SelfAssessment Manikin (SAM) scores of arousal and dominance (Bradley and Lang, 1994). Following the presentation of each image, participants are asked to rate their emotional response ('How negative do you feel?') on a five point Likert scale. On each trial, LOOK or DECREASE cues are presented for $2000 \mathrm{~ms}$, a single IAPS image is then presented for $7000 \mathrm{~ms}$, followed by the emotional response rating $(4000 \mathrm{~ms})$. Each trial is separated by a variable interval of 1000-3000 ms.

Prior to scanning, all participants were given training in emotion regulation and reappraisal strategies using previously validated methods (Garrison et al, 2017). Participants were instructed to think of negative stimuli in ways that made them feel less negative. For example, in the case of an image depicting a funeral, to imagine that someone had died at a very old age after a long and happy life. Ratings of negative affect following each trial type were compared using repeated-measures ANOVA with the between-subjects factor of group and the within subjects factor of condition (Neutral/ Look/Decrease). Due to a technical problem, behavioral data were not recorded for one IGD participant.

\section{Image Acquisition, Spatial Processing, and Subject-Level Statistics}

Functional data were acquired on a Siemens Trio 3T scanner (Siemens AG, Erlangen, Germany) using a $\mathrm{T}^{*}$-sensitive echo-planar image (EPI) gradient-echo pulse sequence (repetition time/echo time $(\mathrm{TR} / \mathrm{TE})=2000 / 25 \mathrm{~ms}$, flip angle $=85^{\circ}$, field-of-view $(\mathrm{FOV})=220 \times 220 \mathrm{~mm}$, matrix $=64 \times$ $64,3.4 \times 3.4 \mathrm{~mm}$ in-plane resolution, slice thickness $=4 \mathrm{~mm}$, 34 slices). Spatial processing, subject-level modeling, and group-level statistics were conducted using SPM12 (Wellcome Functional Imaging Laboratory, London, United
Kingdom). Functional scans were realigned, inspected for excessive motion (scans with displacement $\geqslant 4.5 \mathrm{~mm}$ were excluded) and normalized to Montreal Neurological Institute standard space (voxel size $=3 \times 3 \times 3 \mathrm{~mm}^{3}$ ), prior to smoothing with a $6 \mathrm{~mm}$ full-width-half-maximum Gaussian kernel (Worhunsky et al, 2016). Subject-level models included onsets and durations of events convolved with the hemodynamic response function and motion parameters from realignment, and models were high-pass filtered at $128 \mathrm{~s}$.

\section{Region-of-Interest Analysis}

Based on previous literature (Wilcox et al, 2016), the amygdala was selected as an a priori ROI. A single bilateral amygdala mask was created using the Neuromorphometrics atlas in SPM12 (www.neuromorphometrics.com). Parameter estimates for BOLD signal within this mask were extracted from individual participant maps for each task condition (Neutral/Look/Decrease) and entered into SPSS for betweengroup analyses. Amygdala responses during each viewing condition were compared using a repeated-measures ANOVA with the between-subjects factor of group and the within subjects factor of condition. Post hoc, within-group comparisons of amygdala responses between conditions (eg, Neutral $v s$ Decrease) were Bonferroni corrected for multiple comparisons $(p<0.05)$.

\section{Whole-Brain Analysis}

Whole-brain, between-group differences in BOLD signal were assessed using a single general-linear model (GLM) including group as a between-subject factor and condition (Neutral/Look/Decrease) as a within-subjects factor, using the flexible-factorial module in SPM12. Consistent with current statistical recommendations (Eklund et al, 2016; Flandin and Friston, 2016), whole-brain statistical maps were voxel-level thresholded at $p<0.001$ prior to family-wise error correction ( $\mathrm{pFWE}<0.05)$.

\section{Exploratory Independent Component Analysis}

An ICA was conducted using the GIFT toolbox (http://icatb. sourceforge.net) utilizing the Infomax algorithm (Calhoun et al, 2001b). Further details on ICA analysis methods are provided in Supplementary Materials.

\section{RESULTS}

\section{Self-Reported Affect}

There was a significant main effect of condition on ratings of negative affect $\left(F_{2,43}=146.01, p<0.001\right.$; Supplementary Figure 1). Across all participants, this involved greater ratings of negative affect following all negative images as compared to neutral images, and greater negative affect ratings following the look relative to decrease conditions ( $p$ 's $<0.001)$. However, consistent with prior studies in other addicted populations (Albein-Urios et al, 2013, 2014), ratings of negative affect did not differ between participant groups (interaction of group-by-condition: $F_{2,43}=0.05, p=0.94$ ). 


\section{Amygdala Responses}

Figure 1 shows mean parameter estimates for BOLD responses within the bilateral amygdala ROI across task conditions. Repeated-measures ANOVA indicated a significant group-by-condition interaction $\left(F_{2,44}=6.26, p=0.003\right)$, as well as a main effect of condition $\left(F_{2,44}=9.73, p<0.001\right)$, on BOLD responses within the bilateral amygdala. Across all participants, this involved increased amygdala responses during negative $v s$ neutral affective images ( $p$ 's $\leq 0.002$ ). Follow-up within-group comparisons indicated relatively increased amygdala responses during negative $v s$ neutral affective images $\left(F_{2,21}=30.72, p<0.001\right)$ among control participants, but no differences in amygdala responses between the three task conditions among IGD participants $\left(F_{2,22}=1.18, p=0.33\right)$.

\section{Whole-Brain Analysis}

Whole-brain voxel-wise analyses indicated significant groupby-condition interactions within five clusters (Table 2, Figure 2). The first cluster largely included bilateral cortical regions, including regions of the visual cortex, precuneus cuneus, cingulate, cingulum, supplementary motor cortex, medial and middle frontal gyri, and cerebellum. The second cluster largely encompassed subcortical regions and included the insula, caudate, putamen, thalamus, and amygdala. Additional group-by-condition interactions included clusters involving the right IFG, bilateral middle and superior frontal gyri (MFG and SFG), and middle and superior temporal gyri (MTG and STG).

For the two largest clusters (ie, the bilateral cortical and the subcortical clusters), group-by-condition interactions involved a pattern of increased activation across task conditions among HC participants (ie, Neutral $<$ Look $<$ Decrease), and an opposite pattern of decreasing activation among IGD participants (ie, Neutral $>$ Look $>$ Decrease; Figure 2). For group-bycondition clusters involving the IFG, MFG and SFG, HC participants displayed a similar pattern of increasing activity (ie, Neutral < Look<Decrease), while IGD exhibited relatively unchanged activity across conditions (Figure 2). For the MTG

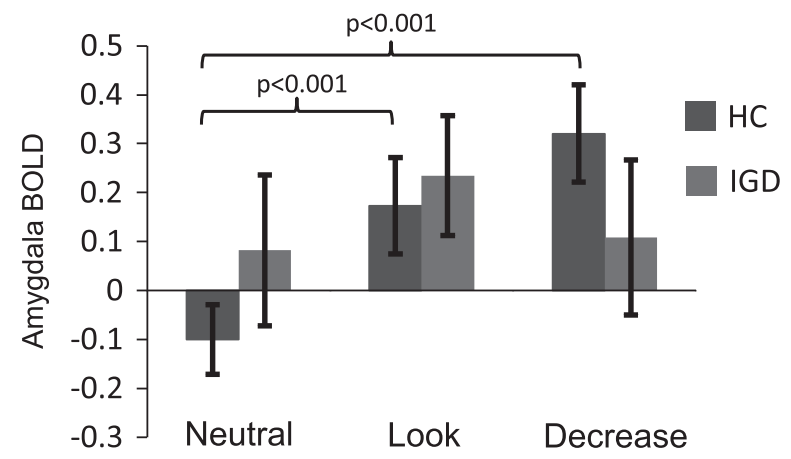

Figure I Amygdala activity during emotion regulation task performance. Shows mean amygdala response (defined using anatomical masks from neuromorphometrics) during each task condition among healthy controls $(\mathrm{HC})$ and individuals with Internet gaming disorder (IGD). Among HCs, amygdala responses were significantly increased while viewing negative images vs neutral images ( $p$ 's $<0.001$ ), however no differences in amygdala responses were found for the maintain vs regulate conditions $(p>0.05$ ). Among IGD participants, no significant differences in amygdala responses were observed across the different viewing conditions ( $p$ 's >0.05). and STG the cluster, the group-by-condition interaction again involved a pattern increasing activity in $\mathrm{HC}$ participants (ie, Neutral $<$ Look $<$ Decrease), whereas IGD participant displayed decreased activity during look relative to neutral and decrease conditions, as well as during neutral relative to decrease conditions (ie, Neutral $>$ Look $<$ Decrease). Group-by-condition interactions for all clusters remained significant following exclusion of IGD participants reporting any alcohol $(n=6)$, tobacco-use $(n=1)$ or both $(n=1)$ left our primary findings unchanged ( $F_{\text {s }}>8.38$., $p$ 's $\left.<0.001\right)$.

Consistent with prior work (Ochsner et al, 2002; Wager et al, 2008; Albein-Urios et al, 2014; Etkin et al, 2015), there were main effects of condition on neural responses with corticolimbic regions including the caudate, thalamus, putamen, insula, IFG, and SFG across all participants (pFWE $<0.05 ;$ Supplementary Figure 2). There were no significant main effects of group on neural responses at whole-brain thresholds ( $\mathrm{pFWE}>0.05)$.

\section{Functional Network Engagement}

Functional inhibitory control networks identified using ICA are shown in Figure 3 (further details in Table 2). Repeatedmeasures ANOVAs confirmed significant main effects of task condition across all participants for engagement of dorsomedial prefrontal $\left(F_{2,44}=18.12, p<0.001\right)$, dorsolateral prefrontal $\left(F_{2,44}=15.99, \quad \mathrm{p}<0.001\right)$ and cingulo-parietal $\left(F_{2,44}=8.06, p=0.002\right)$ networks. For the dorsomedial and dorsolateral prefrontal networks, this involved increased engagement across task conditions (ie, Neutral $<$ Look $<\cap$ Decrease). Whereas for the cingulo-parietal network, this involved relatively decreased engagement across task conditions (ie, Neutral $>$ Look $>$ Decrease). A mixed-effects ANOVA further indicated a significant group-by-condition interaction $\left(F_{2,44}=4.85, p=0.018\right)$ on cingulo-parietal engagement, involving decreased engagement across task conditions among IGD participants, and relatively unchanged activity across conditions among HC participants. This interaction remained significant following exclusion of IGD participants reporting any alcohol or tobacco-use $\left(F_{2,36}=5.19, p=0.012\right)$. No significant group-by-condition interactions were found for engagement of dorsal prefrontal networks (dorsolateral: $F_{2,36}=0.12, p=0.787$ dorsomedial: $\left.\mathrm{F}_{2,36}=0.60, p=0.498\right)$. There were no significant main effects of group on engagement of functional networks ( $p$ 's $>0.05$ ).

\section{Relationships with Clinical Variables}

Within-group Spearman's rho correlations were used to explore relationships between clinical features (trait impulsivity, depressive symptoms and Internet-use severity, as indexed using the Chen Internet Addiction Scale (Chen et al, 2003)) and neural responses within the amygdala (a priori ROI) and within regions or networks identified in interaction analyses (mixed-effects ANOVAs). Given concerns about multiple comparison testing, these analyses were constrained to the Decrease condition only and considered significant at a two-tailed $p<0.01$. Correlational analyses indicated a negative association between Internet-use severity, as indexed by the CIAS (independent measure of Internet-use severity), and subcortical activations (insularstriatal cluster identified in whole-brain ANOVA) during the 
Table 2 Findings from Whole-Brain ANOVA (Voxel-Level $p<0.001$, pFWE $<0.05$ )

\begin{tabular}{|c|c|c|c|c|c|}
\hline \multicolumn{6}{|l|}{ Group by condition interaction } \\
\hline $\begin{array}{l}\text { L \& R thalamus/insula/inferior parietal lobule/superior temporal gyrus/operculum/pulvinar/supramarginal gyrus/precentral } \\
\text { gyrus/inferior frontal gyrus/claustrum/pallidum/parahippocampul gyrus/caudate/putamen/amygdala/L postcentral gyrus }\end{array}$ & 2557 & -18 & -15 & 12 & 31.89 \\
\hline L middle frontal gyrus/superior frontal gyrus & 205 & -33 & 42 & -3 & 31.67 \\
\hline R middle temporal gyrus/superior temporal gyrus & 94 & 60 & -18 & -6 & 23.39 \\
\hline \multicolumn{6}{|l|}{ Independent components (task-relatedness) } \\
\hline \multicolumn{6}{|l|}{ Cingulo-parietal network } \\
\hline \multicolumn{6}{|l|}{ Medial prefrontal network } \\
\hline$L$ \& R medial frontal gyrus/superior frontal gyrus & 633 & -3 & 60 & 24 & 9.26 \\
\hline \multicolumn{6}{|l|}{ Dorsolateral prefrontal network } \\
\hline$L$ inferior frontal gyrus/middle frontal gyrus & 439 & -42 & 48 & 3 & 8.47 \\
\hline R middle frontal gyrus/inferior frontal gyrus/precentral gyrus/SMA & $100 \mid$ & 42 & 6 & 54 & 8.42 \\
\hline L \& R superior frontal gyrus/anterior cingulate/supplementary motor area & 623 & 9 & 9 & 60 & 8.00 \\
\hline
\end{tabular}

Abbreviations: L, left; R, right; SMA, supplementary motor area.

Control Internet gaming disorder
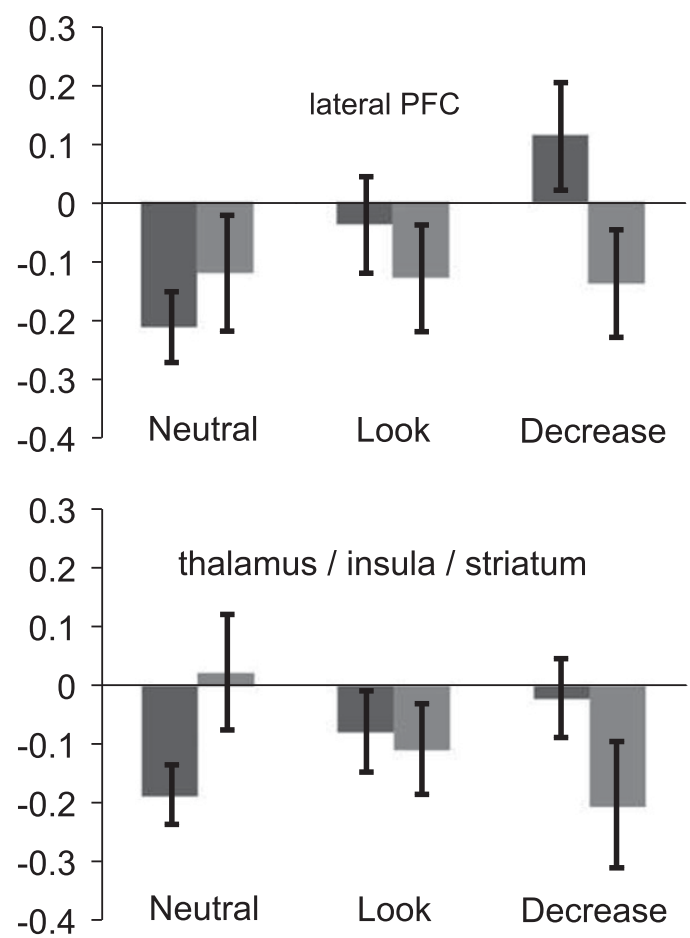

pFWE $<0.05$

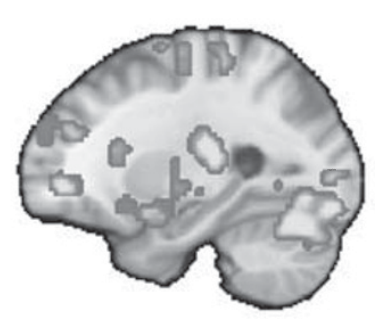

$x=-25$

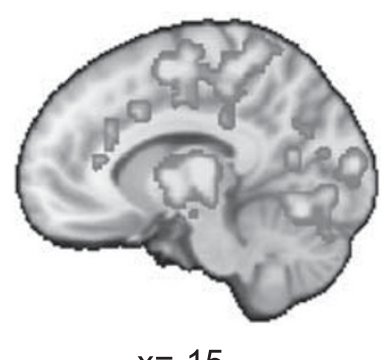

Figure 2 Whole-brain interaction between group (IGD/HC) and task condition (neutral/maintain/regulate). Shows significant group by condition interactions (whole-brain ANOVA; cluster-forming threshold $p<0.001$, pFWE $<0.05$; full results in Table 2) for IGD and HC participants during performance of an emotion regulation task. BOLD activity for selected clusters for each group across conditions are shown for reference. 
a

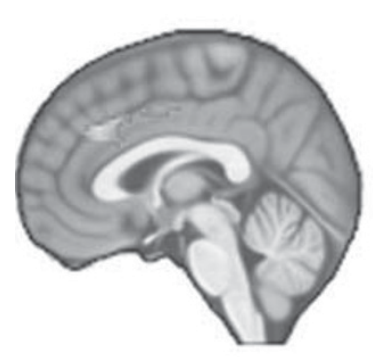

cingulo-parietal IC

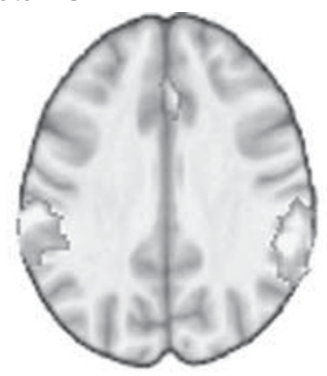

b

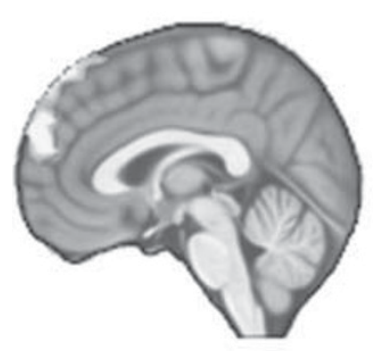

dorsomedial frontal IC
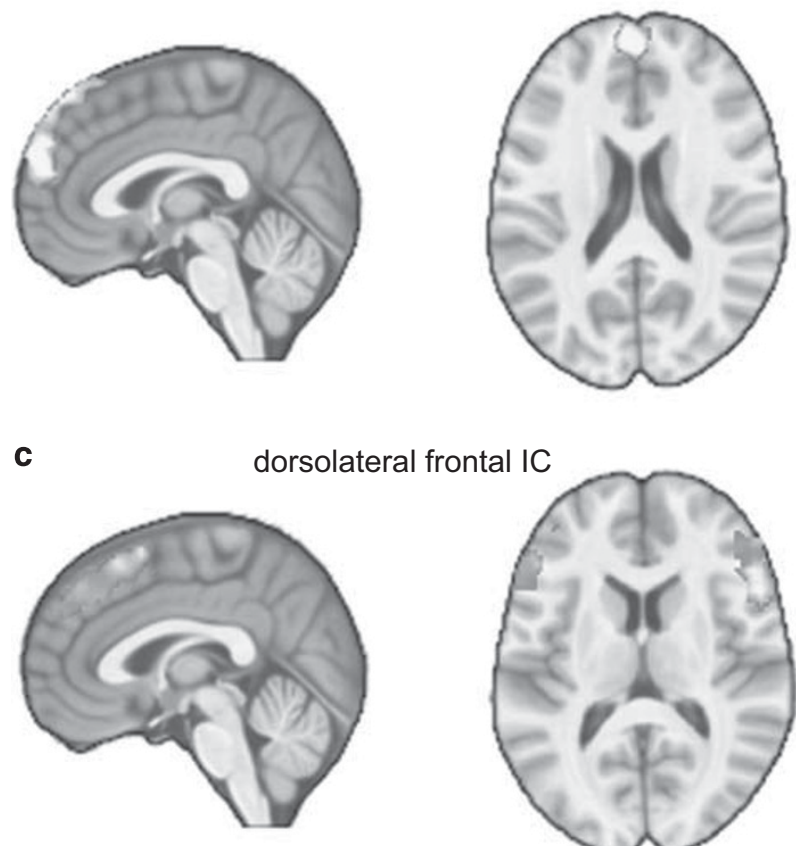

C

Figure 3 Dorsal inhibitory control networks. Shows dorsal inhibitory control networks identified using independent component analysis. Repeated-measures ANOVAs further indicated a significant group by condition interaction $\left(F_{(\mathrm{df}=2)}=4.85, p=0.0\right.$ l 8$)$ on cingulo-parietal network (a) engagement, involving a pattern of decreased engagement during neutral vs maintain vs regulate among IGD participants, and relatively unchanged activity across conditions among HC participants. No significant group by condition interactions were found for engagement of dorsomedial (b) or dorsolateral (c) prefrontal networks ( $p$ 's >0.05).

decrease condition among IGD participants $(r=-0.52$, $p<0.01$; Supplementary Figure 3).

\section{DISCUSSION}

To test the hypothesis that neural processing of negative affect might be blunted among individuals with addictions independent of the effects of drugs of abuse, this study compared neural function during emotion regulation task performance between drug-naïve youth with and without IGD. Consistent with prior work in DUDs (Wang et al, 2010; Smoski et al, 2011; Albein-Urios et al, 2014; Wilcox et al, 2016), individuals with IGD exhibited relatively blunted neural responses to negative affective stimuli within brain regions including the insula, amygdala, striatum and bilateral PFC. Exploratory ICA analyses focusing on inhibitory control networks further indicated alterations in cingulo-parietal network engagement during emotion regulation. To our knowledge, this is the first study of affect processing conducted among drug-naïve individuals with a behavioral addiction, as well as the first affective processing fMRI study of IGD using DSM-5 proposed criteria. The clinical significance of these findings is discussed below.

The amygdala is a critical structure for encoding of threat and negative affect (Ochsner et al, 2012; Etkin et al, 2015). In non-addicted individuals, exposure to negative affective stimuli is associated with increased amygdalar activity (Etkin et al, 2015). Consistent with this, HC participants exhibited increased amygdala activity to negative $v s$ neutral images. In contrast, IGD youth did not demonstrate any differences in amygdala activity across task conditions, despite reporting increased experiences of negative affect. These findings are consistent with other recent reports of blunted amygdala engagement to stress and negative affect among individuals with drug and alcohol use, but suggest that amygdala hypo-reactivity may be a trait feature across drug and behavioral addictions (Wang et al, 2010; Smoski et al, 2011; Wilcox et al, 2016). They further raise the possibilities of both aberrant threat processing and aberrant down-regulatory responses, at least as indexed by amygdalar responsivity, among individuals with addictions (Etkin et al, 2015).

Together with the amygdala, the insula and striatum are critical structures for affective processing (Ochsner et al, 2012; Etkin et al, 2015). In non-addicted individuals, heightened negative affect and difficulties in emotion regulation are typically associated with increased activity, or hyper reactivity, within these regions (Etkin and Wager, 2007; Wilcox et al, 2016). In contrast, emerging data indicate decreased engagement of these regions in response to negative affect among substance-using individuals (AlbeinUrios et al, 2014; Seo et al, 2014; Yip et al, 2016). Consistent with this, individuals with IGD exhibited decreased activity within striatal-insular and thalamic regions in response to negative images. Such decreased engagement of affective neurocircuitry might contribute to the diminished regard for negative consequences exhibited by IGD youth (Yao et al, 2015). Within this context, it is possible that interventions enhancing neural responses to negative feedback might be helpful in reducing gaming behaviors, although further work is needed to test this hypothesis.

Follow-up analyses indicated that, among individuals with IGD, striatal-insular activity was negatively related to addiction severity, such that increased Internet-use severity was associated with greater disengagement during the emotion regulation condition. Recent longitudinal data indicate a positive association between abstinence during a quit attempt and increases in striatal activity in youth with addiction (Garrison et al, 2017). Thus, while the clinical significance of our findings requires further investigation, these data also the possibility that interventions specifically aimed at increasing engagement of limbic reward regions involved in salience encoding, eg, contingency management (Yip et al, 2015; Garrison et al, 2017), might be helpful in reducing Internet addiction severity in youth with IGD. In contrast, recent data suggest that behavioral interventions for IGD may partially operate via a reduction of resting state striatal functional connectivity (Zhang et al, 2016b). Thus, while further work extending treatment-related findings to 
task-based functional connectivity in IGD appears warranted, these data add to a growing body of evidence implicating the insular-striatal regions in the pathophysiology of addictions and their treatment.

Whole-brain analyses further indicated significant group by task condition interactions within bilateral cortical regions, including regions of the lateral PFC previously implicated in 'top-down' inhibitory control and modulation of emotion; eg, the IFG and MFG (Kim and Hamann, 2007; Wager et al, 2008; Drabant et al, 2009). Among control youth, activity within these regions followed the expected pattern of increased engagement to negative images and with increasing task demand (Neutral $<$ Maintain $<$ Regulate). By contrast, IGD youth exhibited relatively blunted prefrontal activity across all task conditions, suggesting a failure to engage lateral prefrontal regions in response to negative affect which may relate to real-world use of reappraisal strategies (Drabant et al, 2009). However, as IGD youth exhibited blunted PFC activity across task conditions, further work is needed to determine the specificity of these findings to emotion regulation, per se.

Exploratory, network-based analyses revealed betweengroup differences in cingulo-parietal network engagement involving decreased engagement across task conditions (ie, Neutral $>$ Look $>$ Decrease), among IGD youth, but relatively unchanged activity across conditions among control youth. Fronto-cingulo-parietal networks dynamically contribute to attention and inhibitory control processes and may be modulated via behavioral and pharmacological interventions (van Amelsvoort and Hernaus, 2016). Prospective associations between cingulo-parietal network engagement and treatment outcomes have been reported in cocaine dependence (Worhunsky et al, 2013) and recent work has demonstrated treatment-associated changes in resting state cingulate functional connectivity among IGD youth (Zhang et al, 2016a). Thus, future studies should explore the specific effects of interventions for IGD on cingulo-parietal network activity during emotion regulatory processes, as this could provide insight into the mechanisms of effective therapies.

Despite the above described differences in neural responses, behavioral ratings of negative affect did not differ between IGD and HC groups. Ratings of negative affect were significantly increased to negative $v s$ neutral stimuli and were decreased following 'Decrease' relative to 'Look' instructions, indicating successful down-modulation of negative affect across all participants. These findings are consistent with a growing body of work suggesting that alterations in neural processing of negative affect may occur independent of conscious awareness among individuals with addictions and with mood disorders (eg, Albein-Urios et al, 2014; Kanske et al, 2015). Within the specific context of this study, the similar behavioral performance across participants may be considered as a strength as it allows us to compare neural activity independent of the behavioral differences that often confound neuroimaging studies (Albein-Urios et al, 2014).

This study has several additional strengths, including the use of a well-validated emotion regulation task in an understudied population-young adults with IGD-and our use of both GLM- and network-based analysis approaches. This is also the first study to assess negative affect among individuals with a behavioral addiction who are in fact drug-naïve. These strengths should nonetheless be considered within the context of several limitations. IGD is not currently a formal DSM-5 diagnosis, but rather a condition for further study (American Psychiatric Association Committee on Nomenclature and Statistics, 2013). Thus, the extent to which our findings may generalize to formal addiction diagnoses remains to be determined. IGD is particularly prevalent among young-adult males (Petry et al, 2015), thus we chose to only include male participants. Further work is therefore needed to extend these findings to young adult females. All youth were drugnaïve, preventing direct comparisons between behavioral and drug addictions. Thus, future research including multiple comparison groups is needed to determine whether these findings extend to youth with substance-use disorders. In addition, given that the development of emotion and regulatory circuitry is ongoing throughout childhood, adolescence and early adulthood (McRae et al, 2012; Yip and Potenza, 2016), further work in different developmental epochs is needed to determine the stability of our findings across development. A final limitation of this study is the absence of an independent self-report measure of emotion regulation (eg, Gross and John, 2003), which might have been more sensitive than within-scanner ratings of negative affect in detecting trait differences in emotion regulation between youth with and without IGD.

This study demonstrates that neural mechanisms subserving negative affect and emotion regulatory processes are altered in an addicted population independent of exposure to drugs of abuse. However, the extent to which alterations in emotional processing might actually precede substance and behavioral addictions, or else might occur as a consequence of repeated activation of endogenous reward circuitry, remains to be determined. In addition, further work directly comparing neural responses between individuals with behavioral and substance addictions are needed to truly test the hypothesis of a shared vs unique neurobiology of addictions (Yip et al, 2017a, b). These data nonetheless add to a growing body of literature indicating that difficulties in emotion regulation in addiction may be linked to hyporesponsivity of limbic regions to negative affective stimuli (Wilcox et al, 2016), and that these alterations might be a common feature of both substance-related and behavioral addictions.

\section{FUNDING AND DISCLOSURE}

This study was supported by NIDA grants K01DA039299 and K12DA000167, the National Center on Addiction and Substance Abuse (CASA) and an Open Project grant from the State Key Laboratory of Cognitive Neuroscience and Learning, National Natural Science Foundation of China (No.81100992), Project of Humanities and Social Sciences supported by Ministry of Education in China (No. 15YJC190035). The authors declare no conflict of interest.

\section{REFERENCES}

Albein-Urios N, Verdejo-Roman J, Asensio S, Soriano-Mas C, Martinez-Gonzalez JM, Verdejo-Garcia A (2014). Re-appraisal of negative emotions in cocaine dependence: dysfunctional corticolimbic activation and connectivity. Addict Biol 19: 415-426. 
Albein-Urios N, Verdejo-Roman J, Soriano-Mas C, Asensio S, Martinez-Gonzalez JM, Verdejo-Garcia A (2013). Cocaine users with comorbid Cluster B personality disorders show dysfunctional brain activation and connectivity in the emotional regulation networks during negative emotion maintenance and reappraisal. Eur Neuropsychopharmacol 23: 1698-1707.

American Psychiatric Association Committee on Nomenclature and Statistics (2013). Diagnostic and Statistical Manual of Mental Disorders5th ednAmerican Psychiatric Association: Washington, DC.

Barnes GM, Welte JW, Hoffman JH, Tidwell M-CO (2009). Gambling, alcohol, and other substance use among youth in the United States. J Stud Alcohol Drugs 70: 134-142.

Beck A, Epstein N, Brown G, Steer RA (1988). An inventory for measuring clinical anxiety: psychometric properties. J Clin Consult Psychol 56: 893-897.

Beck A, Steer R, Brown G (1996). Beck Depression Inventory Manual. The Psychological Corporation: San Antonio, TX.

Bradley MM, Lang PJ (1994). Measuring emotion: the selfassessment manikin and the semantic differential. J Behav Ther Exp Psychiatry 25: 49-59.

Calhoun V, Adali T, Pearlson GD, Pekar JJ (2001a). Spatial and temporal independent component analysis of functional MRI data containing a pair of task-related waveforms. Hum Brain Mapp 13: 43-53.

Calhoun VD, Adali T, Pearlson GD, Pekar JJ (2001b). A method for making group inferences from functional MRI data using independent component analysis. Hum Brain Mapp 14: 140-151.

Chen S, Weng L, Su Y, Wu H, Yang P (2003). Development of a Chinese internet addiction scale and its psychometric study. Chin J Psychol 45: 279-394.

Drabant EM, McRae K, Manuck SB, Hariri AR, Gross JJ (2009). Individual differences in typical reappraisal use predict amygdala and prefrontal responses. Biol Psychiatry 65: 367-373.

Eklund A, Nichols TE, Knutsson H (2016). Cluster failure: why fMRI inferences for spatial extent have inflated falsepositive rates. Proc Natl Acad Sci USA 113: 7900-7905.

Etkin A, Buchel C, Gross JJ (2015). The neural bases of emotion regulation. Nat Rev Neurosci 16: 693-700.

Etkin A, Wager TD (2007). Functional neuroimaging of anxiety: a meta-analysis of emotional processing in PTSD, social anxiety disorder, and specific phobia. Am J Psychiatry 164: 1476-1488.

Flandin G, Friston KJ (2016). Analysis of Family-Wise Error Rates in Statistical Parametric Mapping Using Random Field Theory. University College London, Wellcome Trust Centre for Neuroimaging: UK.

Fox HC, Axelrod SR, Paliwal P, Sleeper J, Sinha R (2007). Difficulties in emotion regulation and impulse control during cocaine abstinence. Drug Alcohol Depend 89: 298-301.

Garrison KA, Yip SW, Balodis IM, Carroll KM, Krishnan-Sarin S, Potenza MN (2017). Reward-related frontostriatal activity and smoking behavior among adolescents in treatment for smoking cessation. Drug Alcohol Depend 177: 268-276.

Gross JJ, John OP (2003). Individual differences in two emotion regulation processes: implications for affect, relationships, and well-being. J Personal Soc Psychol 85: 348-362.

Kanske P, Schonfelder S, Forneck J, Wessa M (2015). Impaired regulation of emotion: neural correlates of reappraisal and distraction in bipolar disorder and unaffected relatives. Transl Psychiatry 5: e497.

Kim SH, Hamann S (2007). Neural correlates of positive and negative emotion regulation. J Cogn Neurosci 19: 776-798.

Lehenbauer-Baum M, Klaps A, Kovacovsky Z, Witzmann K, Zahlbruckner R, Stetina BU (2015). Addiction and engagement: an explorative study toward classification criteria for internet gaming disorder. Cyberpsychol Behav Soc Netw 18: 343-349.
Liu L, Yip SW, Zhang JT, Wang LJ, Shen ZJ, Liu B et al (2017). Activation of the ventral and dorsal striatum during cue reactivity in Internet gaming disorder. Addict Biol 22: 791-801.

McRae K, Gross JJ, Weber J, Robertson ER, Sokol-Hessner P, Ray RD et al (2012). The development of emotion regulation: an fMRI study of cognitive reappraisal in children, adolescents and young adults. Soc Cogn Affect Neurosci 7: 11-22.

Meda SA, Gill A, Stevens MC, Lorenzoni RP, Glahn DC, Calhoun $\mathrm{VD}$ et al (2012). Differences in resting-state functional magnetic resonance imaging functional network connectivity between schizophrenia and psychotic bipolar probands and their unaffected first-degree relatives. Biol Psychiatry 71: 881-889.

Mihara S, Higuchi S (2017). Cross-sectional and longitudinal epidemiological studies of Internet gaming disorder: a systematic review of the literature. Psychiatry Clin Neurosci 71: 425-444.

Ochsner KN, Bunge SA, Gross JJ, Gabrieli JD (2002). Rethinking feelings: an FMRI study of the cognitive regulation of emotion. $J$ Cogn Neurosci 14: 1215-1229.

Ochsner KN, Silvers JA, Buhle JT (2012). Functional imaging studies of emotion regulation: a synthetic review and evolving model of the cognitive control of emotion. Ann N Y Acad Sci 1251: E1-24.

Patton JH, Stanford MS, Barratt ES (1995). Factor structure of the barratt impulsiveness scale. J Clin Psychol 51: 768-774.

Peters EN, Nordeck C, Zanetti G, O'Grady KE, Serpelloni G, Rimondo C et al (2015). Relationship of gambling with tobacco, alcohol, and illicit drug use among adolescents in the USA: review of the literature 2000-2014. Am J Addict 24: 206-216.

Petry NM, Rehbein F, Gentile DA, Lemmens JS, Rumpf HJ, Mossle $\mathrm{T}$ et al (2014). An international consensus for assessing internet gaming disorder using the new DSM-5 approach. Addiction 109: 1399-1406.

Petry NM, Rehbein F, Ko CH, O'Brien CP (2015). Internet gaming disorder in the DSM-5. Curr Psychiatry Rep 17: 72.

Rehbein F, Kliem S, Baier D, Mossle T, Petry NM (2015). Prevalence of internet gaming disorder in German adolescents: diagnostic contribution of the nine DSM-5 criteria in a state-wide representative sample. Addiction 110: 842-851.

Seo D, Lacadie CM, Tuit K, Hong KI, Constable RT, Sinha R (2013). Disrupted ventromedial prefrontal function, alcohol craving, and subsequent relapse risk. JAMA Psychiatry 70: 727-739.

Seo D, Tsou KA, Ansell EB, Potenza MN, Sinha R (2014). Cumulative adversity sensitizes neural response to acute stress: association with health symptoms. Neuropsychopharmacology 39: 670-680.

Sheehan D, Lecrubier Y, Sheehan K, Amorim P, Janavs J, Weiller E et al (1998). The Mini-International Neuropsychiatric Interview (M.I.N.I.): the development and validation of a structured diagnostic psychiatric interview for DSM-IV and ICD-10. J Clin Psychiatry 59(Suppl 20): 22-33.

Si T-M, Shu L, Dang W-M, Se Y-A, Chen J-X, Dong W-T et al (2009). Evaluation of the reliability and validity of Chinese version of the Mini-International Neuropsychiatric Interview in patients with mental disorders. Chin Ment Health J 23: 493-503. Smith SM, Miller KL, Moeller S, Xu J, Auerbach EJ, Woolrich MW et al (2012). Temporally-independent functional modes of spontaneous brain activity. Proc Natl Acad Sci 109: 3131-3136.

Smoski MJ, Salsman N, Wang L, Smith V, Lynch TR, Dager SR et al (2011). Functional imaging of emotion reactivity in opiatedependent borderline personality disorder. Personal Disord 2: 230-241.

van Amelsvoort T, Hernaus D (2016). Effect of pharmacological interventions on the fronto-cingulo-parietal cognitive control network in psychiatric disorders: a transdiagnostic systematic review of fMRI studies. Front Psychiatry 7: 82.

Volkow ND, Koob GF, McLellan AT (2016). Neurobiologic advances from the brain disease model of addiction. $N$ Engl J Med 374: 363-371. 
Wager TD, Davidson ML, Hughes BL, Lindquist MA, Ochsner KN (2008). Neural mechanisms of emotion regulation: evidence for two independent prefrontal-subcortical pathways. Neuron 59: 1037-1050.

Wang ZX, Zhang JX, Wu QL, Liu N, Hu XP, Chan RC et al (2010). Alterations in the processing of non-drug-related affective stimuli in abstinent heroin addicts. Neuroimage 49: 971-976.

Weinstein A, Livny A, Weizman A (2017). New developments in brain research of internet and gaming disorder. Neurosci Biobehav Rev 75: 314-330.

Wilcox CE, Pommy JM, Adinoff B (2016). Neural circuitry of impaired emotion regulation in substance use disorders. Am J Psychiatry 173: 344-361.

Worhunsky PD, Dager AD, Meda SA, Khadka S, Stevens MC, Austad CS et al (2016). A preliminary prospective study of an escalation in 'Maximum Daily Drinks', fronto-parietal circuitry and impulsivity-related domains in young adult drinkers. Neuropsychopharmacology 41: 1637-1647.

Worhunsky PD, Stevens MC, Carroll KM, Rounsaville BJ, Calhoun VD, Pearlson GD et al (2013). Functional brain networks associated with cognitive control, cocaine dependence, and treatment outcome. Psychol Addict Behav 27: 477-488.

Xu J, Potenza MN, Calhoun VD (2013a). Spatial ICA reveals functional activity hidden from traditional fMRI GLM-based analyses. Front Neurosci 7: 154.

Xu J, Potenza MN, Calhoun VD (2013b). Spatial ICA reveals functional activity hidden from traditional fMRI GLM-based analyses. Front Neurosci 7: 154.

Xu J, Potenza MN, Calhoun VD, Zhang R, Yip SW, Wall JT et al (2016). Large-scale functional network overlap is a general property of brain functional organization: reconciling inconsistent fMRI findings from general-linear-model-based analyses. Neurosci Biobehav Rev 71: 83-100.

Yao YW, Chen PR, Li S, Wang LJ, Zhang JT, Yip SW et al (2015). Decision-making for risky gains and losses among college students with Internet Gaming Disorder. PLoS ONE 10: e0116471.

Yip S, Carroll K, Potenza M (2015). An overview of translational approaches to the treatment of addictions. In: Feldstein Ewing S, Witkiewitz K, Filbey F (eds). Neuroimaging and Psychosocial
Addiction Treatment: An Integrative Guide for Researchers and Clinicians. Palgrave: New York, NY.

Yip SW, Desai RA, Steinberg MA, Rugle L, Cavallo DA, KrishnanSarin S et al (2011). Health/functioning characteristics, gambling behaviors, and gambling-related motivations in adolescents stratified by gambling problem severity: findings from a high school survey. Am J Addict 20: 495-508.

Yip SW, Lacadie CM, Sinha R, Mayes LC, Potenza MN (2016). Prenatal cocaine exposure, illicit-substance use and stress and craving processes during adolescence. Drug Alcohol Depend 158: 76-85.

Yip SW, Morie KM, Xu J, Constable RT, Malison RT, Carroll KM et al (2017a). Shared microstructural features of behavioral and substance addictions revealed in areas of crossing fibers. Biol Psychiatry: Cogn Neurosci Neuroimaging 2: 188-195.

Yip SW, Potenza MN (2016). Application of Research Domain Criteria to childhood and adolescent impulsive and addictive disorders: implications for treatment. Clin Psychol Rev; doi: 10.1016/j.cpr.2016.11.003 [e-pub ahead of print].

Yip SW, Worhunsky PD, Xu J, Morie KP, Constable RT, Malison RT et al (2017b). Gray-matter relationships to diagnostic and transdiagnostic features of drug and behavioral addictions. Addict Biol; doi: 10.1111/adb.12492 [e-pub ahead of print].

Young KS (2009). Internet Addiction Test (IAT). http://netaddic tion.com/index.php?option $=$ combfquiz\&view $=$ onepage $\&$ catid $=$ 46\&Itemid $=106$.

Zhang J-T, Yao Y-W, Potenza MN, Xia C-C, Lan J, Liu L et al (2016a). Altered resting-state neural activity and changes following a craving behavioral intervention for Internet gaming disorder. Sci Rep 6: 28109.

Zhang JT, Ma SS, Li CR, Liu L, Xia CC, Lan J et al (2016b). Craving behavioral intervention for internet gaming disorder: remediation of functional connectivity of the ventral striatum. Addict Biol; doi: 10.1111/adb.12474 [e-pub ahead of print].

Zhang JT, Yao YW, Li CS, Zang YF, Shen ZJ, Liu L et al (2016c). Altered resting-state functional connectivity of the insula in young adults with Internet gaming disorder. Addict Biol 21: 743-751.

Supplementary Information accompanies the paper on the Neuropsychopharmacology website (http://www.nature.com/npp) 